\title{
Augmented EPA with Augmented EFIE Method for Packaging Analysis
}

\author{
Zuhui Ma ${ }^{\# 1}$, Lijun Jiang ${ }^{\# 2}$, Wengcho Chew ${ }^{\# * 3}$, Maokun Li ${ }^{\$ 4}$, Zhiguo Qian ${ }^{* 5}$ \\ ${ }^{*}$ Dept. Of Electrical and Electronic Engineering, the University of Hong Kong \\ Chow Ye Ching Building, the University of Hong Kong, Pokfulam, HK \\ ${ }^{1}$ zuhuima@gmail.com, ${ }^{2}$ ljiang@eee.hku.hk, ${ }^{3}$ wcchew@hku.hk \\ *Dept. Of Electrical Engineering, the University of Illinois at Urbana-Champaign, USA \\ ${ }^{3}$ wcchewduiuc.edu \\ ${ }^{\$}$ Schlumberger-Doll Research, Cambridge, USA \\ ${ }^{4}$ maokunliegmail.com
}

\begin{abstract}
It is evident that the low frequency full wave electromagnetic modelling is necessary for IC packaging analysis. Considering the complexity, it is very difficult to solve the whole problem directly. Even though the domain decomposition method is a legitimate approach for these types of problems, the domain decomposition method based on the equivalence principle has the low frequency breakdown issue. In this paper, we developed a low frequency augmented equivalence principle algorithm (AEPA) with the augmented electric field integral equation (AEFIE) for packaging and IC analysis. On the equivalence surfaces, not only the electric current and the magnetic current, but also the electric charge and the magnetic charge are used to capture the low frequency couplings. Inside each AEPA box, AEFIE is applied to maintain the low frequency accuracy. As a result, we are able to solve low frequency domain decomposition problems and apply it to IC packaging analysis.
\end{abstract}

\section{INTRODUCTION}

Practical complex structures requiring electromagnetic (EM) analysis frequently have the multiscale and multiphysics features [1]. Low frequency EM modeling is critical to today's IC industries, especially the physical layout design. The solutions of these problems are instrumental in the modelling of complex electronic package structures found in modern integrated circuit design, small antennas, and small sensors. Furthermore, they will find applications in the modelling of micro and nano structures in nano-technology and nano-biotechnology. The regime of low-frequency problems is where circuit physics dominates over wave physics. The physics of electromagnetic fields is quite different in this regime compared to higher frequency electromagnetic fields $[2,3]$.

A popular way of solving such problems is to use integral equation methods [4]. However, integral equation methods have low frequency breakdown. This is because that at low frequencies, the electric field and the magnetic field are weakly coupled, compared to the wave-physics regime. Hence, most numerical methods designed do not capture these two physical phenomena well and hence, the breakdown. To solve the low frequency problem in integral equation methods, loop-tree method was broadly used because the current decomposition satisfies the circuit physics at low frequency
[5]. However, the loop-tree basis generation is a big trouble. It could be even slower than the integral equation solver itself.

Recently Qian and Chew developed the augmented electric field integral equation (AEFIE) method [6] which uses only RWG basis [7] and patch basis. In this method, the EFIE is augmented with an additional charge unknown, and an additional continuity equation relating the charge to the current. The resultant equation, after proper frequency normalization, is frequency stable down to very low frequency. This method apparently does not suffer from the lowfrequency breakdown, but it does have the low-frequency inaccuracy problem, which was later solved through the perturbation method [8].

However, it is still not feasible to solve the practical full system, such as the whole packaging or PCB board. The resource usage demands high performance computing (HPC) for the algorithm development and relevant hardware support. To overcome this problem, a strict multi-scale domain decomposition method - equivalence principle algorithm (EPA) was developed [9]. It uses the equivalent electric current and magnetic current to reconstruct the field. Hence, it turns the interaction between sub-domain details into the interaction between sub-domain surfaces, which greatly reduced the simulation cost [10]. However, there is a low frequency breakdown issue with EPA [11]. From the evidence we will show in this paper, EPA method breaks down when the sub-domain is relatively small compared to the wavelength. This greatly limited the applications of this powerful method, especially to low frequency problems, such as IC packaging and on-chip interconnects.

This paper focuses on understanding the low frequency breakdown issue of the EPA method and discusses how to solve this problem. We introduce equivalent electric charges and magnetic charges on equivalent surfaces of EPA to formulate the augmented EPA method. Different from the previous effort made by Sun and Chew [11], to solve the low frequency breakdown happening inside each EPA box, the augmented EFIE instead of the loop-tree method is applied to replace the conventional EPA scattering operator. Hence, the low frequency physics is well maintained inside and outside 
the EPA box. The benchmarks shown at the end of this paper will demonstrate the validity of the proposed method.

\section{AUGMENTED EFIE}

Recently, a stable electric field integral equation (EFIE) using an augmentation technique [6], [8] was developed. This method treats the charge as an unknown and introduces an additional equation to relate the current and the charge. The final matrix equation has the generalized saddle point form. This technique avoids the use of loop-tree decomposition, and provides a stable EFIE formulation down to very low frequencies. The search for loops and trees of a highly complex structure is often very challenging. This method, obviating that need, heralds a new way of solving lowfrequency electromagnetic, complex structure, problems with multi-scale features. In this method, the electric field integral equation (EFIE) can be written in a matrix form as

$$
\left(i k_{0} \eta_{0} \overline{\mathbf{V}}+\frac{\eta_{0}}{i k_{0}} \overline{\mathbf{S}}\right) \cdot \mathbf{J}=\mathbf{b}
$$

where

$$
\begin{aligned}
& {\left[\overline{\mathbf{V}} \lambda_{m, n} \mathbf{r}=\boldsymbol{A} \boldsymbol{n}_{r} \int_{S_{m}} \mathbf{r} \mathbf{r}_{m}() \cdot \int_{S_{n}} g\left(,^{\prime}\right){ }_{n}\left({ }^{\prime}\right) d S^{\prime} d S\right.} \\
& {\left[\overline{\mathbf{S}} \lambda_{m, n} \mathbf{r}=\mathbf{A r}_{r}^{-1} \int_{S_{m}} \mathbf{r} \cdot{ }_{m}() \int_{S_{n}} g\left(,^{\prime}\right) \nabla^{\prime} \cdot{ }_{n}\left({ }^{\prime}\right) d S^{\prime} d S\right.} \\
& {[\overline{\mathbf{P}}]_{m, n}=\varepsilon_{r}^{-1} \int_{T_{m}} h_{m}(\mathbf{r}) \int_{T_{n}} g\left(\mathbf{r}, \mathbf{r}^{\prime}\right) h_{n}\left(\mathbf{r}^{\prime}\right) d S^{\prime} d S}
\end{aligned}
$$

In the above, $\boldsymbol{\Lambda} \mathbf{r}($ ) is the RWG basis function [7]. The scalar potential matrix $\overline{\mathbf{S}}$ can be factorized as

$$
\overline{\mathbf{S}}=\overline{\mathbf{D}}^{T} \cdot \overline{\mathbf{P}} \cdot \overline{\mathbf{D}}
$$

where $\overline{\mathbf{D}}$ has the meaning of a divergence operator. The current continuity condition yields

$$
\overline{\mathbf{D}} \cdot \boldsymbol{\phi}=i k_{0} c_{0}
$$

where $c_{0}$ is the speed of light.

Combining (6), (3), and (4), we get the A-EFIE as

$$
\left[\begin{array}{cc}
\overline{\mathbf{V}} & \overline{\mathbf{D}}^{T} \cdot \overline{\mathbf{P}} \\
\overline{\mathbf{D}} & k_{0}^{2} \overline{\mathbf{I}}
\end{array}\right] \cdot\left[\begin{array}{c}
i k_{0} \mathbf{J} \\
c_{0} \boldsymbol{\rho}
\end{array}\right]=\left[\begin{array}{c}
\eta_{0}^{-1} \mathbf{b} \\
\mathbf{0}
\end{array}\right]
$$

where $\overline{\mathbf{I}} \in \mathrm{R}^{p \times p}$ is an identity matrix, and $\boldsymbol{\rho} \in \mathrm{C}^{p \times 1}$ is the vector of charge coefficient. The matrix has a $2 \times 2$ block structure. More details about this work can be found in reference [6].

\section{EQUIVALENCE PRINCIPAL ALGORITHM}

The equivalence principle algorithm (EPA) is a good way to domain-decompose a larger problem into smaller problems [9]-[10]. It also allows regions of low frequency physics (circuit physics) to be separated from the regions of mid frequency physics (wave physics). The use of EPA allows a larger problem to be broken down into a sum of smaller problems, so that only smaller problems need to be solved at one time. Then the solution to the larger problem is accomplished by rigorously concatenating the smaller problems together.

EPA also allows one to use one technique to solve the smaller problems, and a different technique for the larger problem. This is important since the physics at the microscale is quite different from the physics at the macro-scale.
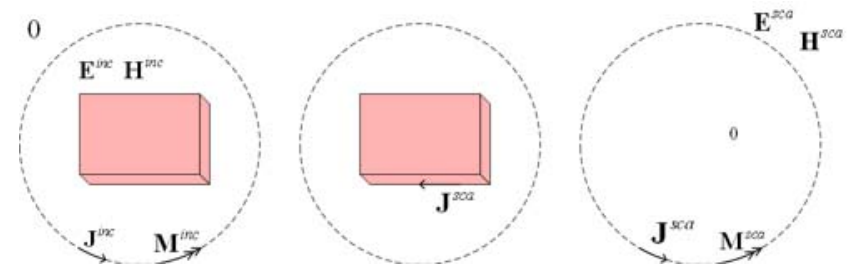

Figure 1. (Left) The incident field is propagated onto the scatterer using equivalence currents on the equivalence surface. (Middle) The current on the scatterer due to incident field is solved for using MOM. (Right) The scattered field is transmitted to infinity using equivalence currents on the equivalence surface again.

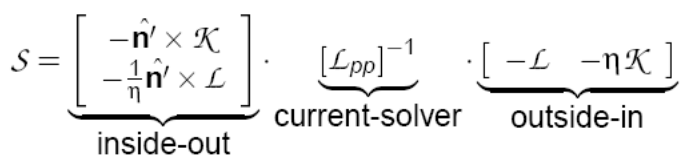

The physics of the scattering is encapsulated in the equivalence surfaces. The incident field from outside is first generated by equivalence currents on the equivalence surface (Figure 1). Then the current induced on the scatterer is found by MOM, and the scattered field is first used to generate equivalence currents on the equivalence surface. These equivalence currents can be used to find the scattered field everywhere outside. In this manner, a scattering operator can be defined.

Then interactions between multiple objects need to be accounted for. They can be done using the translation operator which finds equivalence currents on one equivalence surface due to equivalence currents on another equivalence surface. The translation operator is defined as:

$T^{h h \cdot} \cdot\left[\begin{array}{c}\mathbf{J}_{1} \\ \frac{1}{\eta} \mathbf{M}_{1}\end{array}\right]=\left[\begin{array}{cc}-\hat{\mathbf{n}} \times \mathcal{K}_{H J}^{S} & -\frac{1}{\eta} \hat{\mathbf{n}} \times \mathcal{L}_{H M}^{S} \\ -\frac{1}{\eta} \hat{\mathbf{n}} \times \mathcal{L}_{E J}^{S} & -\hat{\mathbf{n}} \times \mathcal{K}_{E M}^{S}\end{array}\right] \cdot\left[\begin{array}{c}\mathbf{J}_{1} \\ \frac{1}{\eta} \mathbf{M}_{1}\end{array}\right]$

Consequently, when multiple objects are interacting with each other, their interactions can be described by the scattering operators and translation operators defined above (Figure 2). For instance, the interaction between three objects can be described as:

$$
\begin{gathered}
{\left[\begin{array}{c}
\mathbf{J}_{1}^{s c a} \\
\frac{1}{\eta} \mathbf{M}_{1}^{s c a}
\end{array}\right]-S_{11} \cdot \mathcal{T}_{12}^{h h} \cdot\left[\begin{array}{c}
\mathbf{J}_{2}^{s a c} \\
\frac{1}{\eta} \mathbf{M}_{2}^{s c a}
\end{array}\right]-S_{11} \cdot \mathcal{T}_{13}^{h p} \cdot \mathbf{J}_{3}=S_{11} \cdot\left[\begin{array}{c}
\mathbf{J}_{1}^{\text {hic }} \\
\frac{1}{\eta} \mathbf{M}_{1}^{\text {inc }}
\end{array}\right]} \\
-S_{22} \cdot \mathcal{T}_{21}^{h h} \cdot\left[\begin{array}{c}
\mathbf{J}_{1}^{s a c} \\
\frac{1}{\eta} \mathbf{M}_{1}^{s c a}
\end{array}\right]+\left[\begin{array}{c}
\mathbf{J}_{2}^{s a c} \\
\frac{1}{\eta} \mathbf{M}_{2}^{s c a}
\end{array}\right]-S_{22} \cdot \mathcal{T}_{23}^{h p} \cdot \mathbf{J}_{3}=S_{22} \cdot\left[\begin{array}{c}
\mathbf{J}_{2}^{i n c} \\
\frac{1}{\eta} \mathbf{M}_{2}^{i n c}
\end{array}\right] \\
\mathcal{T}_{31}^{p h} \cdot\left[\begin{array}{c}
\mathbf{J}_{1}^{s c a} \\
\frac{1}{\eta} \mathbf{M}_{1}^{s c a}
\end{array}\right]+\mathcal{T}_{32}^{p h} \cdot\left[\begin{array}{c}
\mathbf{J}_{2}^{s c a} \\
\frac{1}{\eta} \mathbf{M}_{2}^{s c a}
\end{array}\right]+\mathcal{L}_{33}^{s} \cdot \mathbf{J}_{3}=-\mathbf{E}_{3}^{i n c}
\end{gathered}
$$




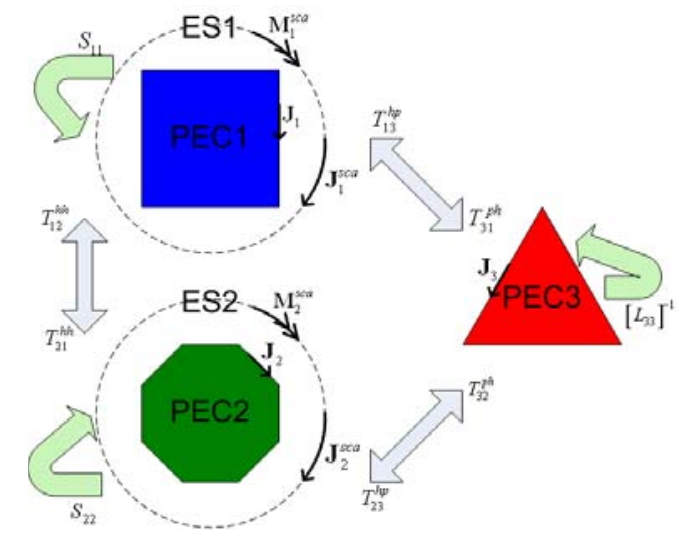

Figure 2. Multiple objects can be concatenated by using EPA.

\section{LOW FREQUENCY BREAKDOWN OF EPA}

Through benchmarks, it is found that EPA method has been working very well for radiating problems, but will divergence when the frequency goes down [11]. To demonstrate this issue, an inductive loop was simulated using EPA method. In Figure 3, the inductive loop was analysed using AEFIE, EFIE and EPA methods respectively. The magnitudes of calculated admittances are shown in Figure 4. Because AEFIE has the proven low frequency accuracy, we use its data as the reference. Its result also shows the correct linear scaling property of the loop inductance vs. the frequency. Because of the well known low frequency breakdown issue, EFIE result diverges when frequency goes low. We also see that EPA diverges when the frequency is low.

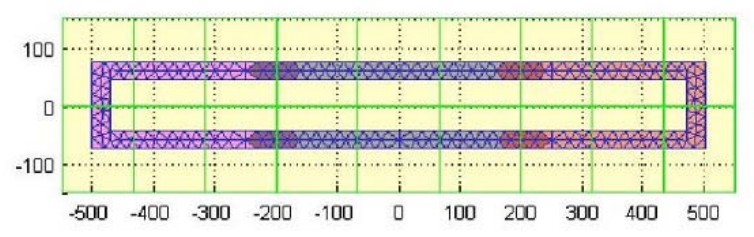

Figure 3. An inductive loop being simulated by EPA method.

The reason of this low frequency breakdown issue for EPA comes from two reasons:

1) The Scattering Operator: Each EPA box will be electrically small when the frequency goes low. Hence, the three step scattering process (scattering operator) is dominated by the low frequency circuit physics. Hence, the conventional RWG based method will not correctly reflect the Helmholtz decomposition happening at the low frequency regime. As a result, EPA will eventually lose the accuracy.

2) Translation Operator: The translation operators are in charge of inter-EPA box interactions. When the frequency goes low, they will also fall into the low frequency regime. Hence, the previous representation of equivalent electric current and magnetic current will not be enough to deal with the circuit physics. They will also contribute to the bad accuracy at low frequencies.

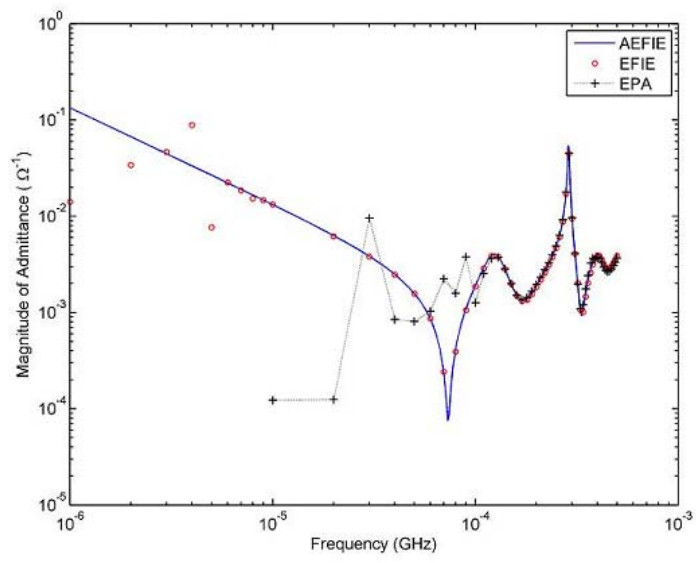

Figure 4. The admittance results of the inductive loop simulated using AEFIE, EFIE, and EPA method.

\section{AugMented EPA with AugMENTEd EFIE}

Considering the abovementioned analysis, to solve the first issue, it will be natural to replace the scattering operator with AEFIE operator so that the internal low frequency breakdown issue can be avoided. To be more specific, the current solver operator $L_{p p}$ will be replaced by AEFIE solver. The result was promising but did not completely remove the problem.

To make EPA method completely comply with the low frequency, we introduced electric charges and magnetic charges on the surface of equivalence surfaces. The scattering operator is changed into

$$
\mathcal{S}=\left[\begin{array}{c}
-\hat{\mathbf{n}}^{\prime} \times \mathcal{K}_{H J}^{S} \\
-\frac{1}{\eta} \hat{\mathbf{n}}^{\prime} \times \mathcal{L}_{E J}^{S} \\
\frac{1}{c_{0} \eta} \hat{\mathbf{n}}^{\prime} \cdot \mathcal{L}_{E J}^{s} \\
-\frac{\eta}{c_{0}} \hat{\mathbf{n}}^{\prime} \cdot \mathcal{K}_{H J}^{s}
\end{array}\right] \cdot\left[\mathcal{L}_{p p]}\right]_{\mathrm{A}-\mathrm{EFIE}}^{-1} \cdot\left[-\mathcal{L}_{E J, v}^{S},-\eta \mathcal{K}_{E M}^{S},-\mathcal{L}_{E J, s}^{S}, 0\right]
$$

If we still consider the object configuration shown in Figure 2 , the interaction between objects represented by Equation (8) will be changed to

$$
\begin{aligned}
& {\left[\begin{array}{c}
\mathbf{J}_{1}^{s c a} \\
\frac{1}{\eta} \mathbf{M}_{1}^{s c a} \\
\boldsymbol{\rho}_{1}^{e} \\
\boldsymbol{\rho}_{1}^{m}
\end{array}\right]-\tilde{\mathcal{S}}_{11} \cdot \tilde{\mathcal{T}}_{12}^{h h} \cdot\left[\begin{array}{c}
\mathbf{J}_{2}^{s c a} \\
\frac{1}{\eta} \mathbf{M}_{2}^{s c a} \\
\rho_{2}^{e} \\
\rho_{2}^{m}
\end{array}\right]-\tilde{\mathcal{S}}_{11} \cdot \tilde{\mathcal{T}}_{13}^{h p} \cdot\left[\begin{array}{c}
\mathbf{J}_{3} \\
\boldsymbol{\rho}_{3}
\end{array}\right]=\tilde{\mathcal{S}}_{11} \cdot\left[\begin{array}{c}
\mathbf{J}_{1}^{\text {inc }} \\
\frac{1}{\eta} \mathbf{M}_{1}^{\text {inc }} \\
\boldsymbol{\rho}_{1}^{e, \text { inc }} \\
\boldsymbol{\rho}_{1}^{m, i n c}
\end{array}\right]} \\
& -\tilde{\mathcal{S}}_{22} \cdot \tilde{\mathcal{T}}_{21}^{h h} \cdot\left[\begin{array}{c}
\mathbf{J}_{1}^{s c a} \\
\frac{1}{\eta} \mathbf{M}_{1}^{s c a} \\
\boldsymbol{\rho}_{1}^{e} \\
\boldsymbol{\rho}_{1}^{m}
\end{array}\right]+\left[\begin{array}{c}
\mathbf{J}_{2}^{s c a} \\
\frac{1}{\eta} \mathbf{M}_{2}^{s c a} \\
\rho_{2}^{e} \\
\rho_{2}^{m}
\end{array}\right]-\tilde{\mathcal{S}}_{22} \cdot \tilde{\mathcal{T}}_{23}^{h p} \cdot\left[\begin{array}{c}
\mathbf{J}_{3} \\
\rho_{3}
\end{array}\right]=\tilde{\mathcal{S}}_{22} \cdot\left[\begin{array}{c}
\mathbf{J}_{2}^{\text {inc }} \\
\frac{1}{\eta} \mathbf{M}_{2}^{\text {inc }} \\
\rho_{2}^{e, i n c} \\
\rho_{2}^{m, i n c}
\end{array}\right] \\
& \tilde{\mathcal{T}}_{31}^{p h} \cdot\left[\begin{array}{c}
\mathbf{J}_{1}^{s c a} \\
\frac{1}{\eta} \mathbf{M}_{1}^{s c a} \\
\boldsymbol{\rho}_{1}^{e} \\
\boldsymbol{\rho}_{1}^{m}
\end{array}\right]+\tilde{\mathcal{T}}_{32}^{p h} \cdot\left[\begin{array}{c}
\mathbf{J}_{2}^{s c a} \\
\frac{1}{\eta} \mathbf{M}_{2}^{s c a} \\
\rho_{2}^{e} \\
\rho_{2}^{m}
\end{array}\right]+\tilde{\mathcal{L}}_{33}^{S} \cdot\left[\begin{array}{c}
\mathbf{J}_{3} \\
\rho_{3}
\end{array}\right]=-\mathbf{E}_{3}^{i n c}
\end{aligned}
$$

It is seen that the electric and magnetic charge distributions are solved together with the current distributions. They will compensate the accuracy loss due to the circuit physics at low frequencies. 


\section{NUMERICAL RESULTS}

The scattering of a metallic unit sphere was studies using (1) conventional EPA method; (2) AEPA with AEFIE method. The data are shown in Figure 5 and 6 . They demonstrate that EPA method will diverge at $1 \mathrm{MHz}$ while AEPA with AEFIE can simulate even the $2 \mathrm{KHz}$ low frequency problem.

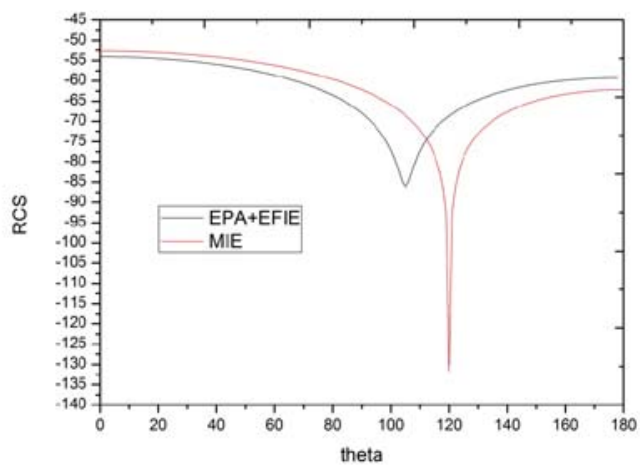

Figure 5. Scattering of a unit sphere simulated by EPA with EFIE at 1MHz.

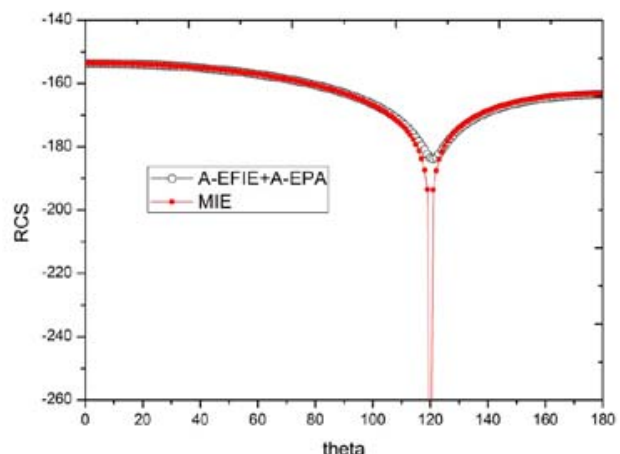

Figure 6. Scattering of a unit sphere simulated by AEPA with AEFIE at 2KHz.

A BGA packaging solder ball array was modeled and simulated by the new method. One ball is excited as the feed while the coupling current distribution was analyzed. Because all the balls have the similar geometry, AEPA with AEFIE only needs to solve one ball's scattering operator and reuse it for coupling interactions. The current distribution is shown in Figure 7. The used frequency is $300 \mathrm{MHz}$.

\section{CONCLUSIONS}

In this paper, a novel augmented EPA method with augmented EFIE method is introduced to solve low frequency domain decomposition problems. Equivalent surface electric charges and surface magnetic charges are applied to EPA to solve the low frequency breakdown. AEFIE is used to solve the scattering operator. From the numerical verification, the proposed method is able to solve very low frequency problems for packaging structures effectively.

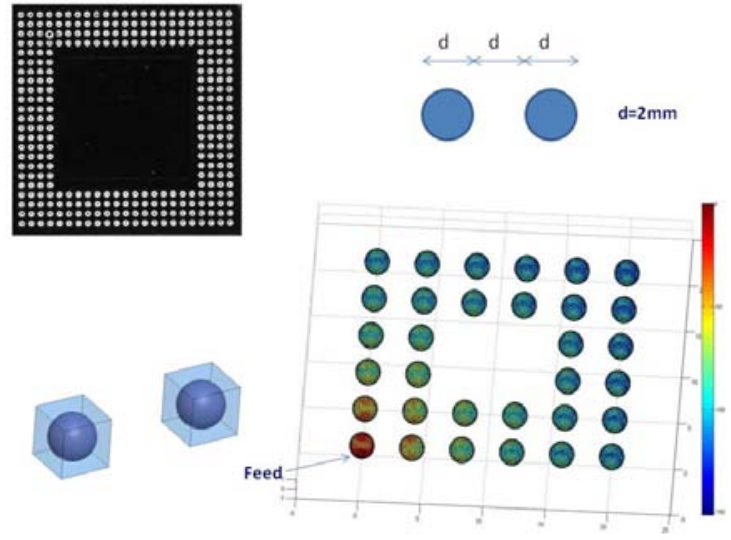

Figure 8. BGA ball array analysis at $300 \mathrm{MHz}$

\section{ACKNOWLEDGMENT}

We sincerely acknowledge the support of Hong Kong GRF Funding for this work.

\section{REFERENCES}

[1] W. C. Chew, L.J. Jiang, Y.H. Chu, G.L. Wang, I.T. Chiang, Y.C. Pan, and J.S. Zhao, "Toward a More Robust and Accurate CEM Fast Integral Equation Solver for IC Applications," IEEE Trans. Advanced Packaging, vol. 28, no. 3, pp. 449-464, Aug. 2005

[2] W. C. Chew, "Computational Electromagnetics---the Physics of Smooth versus Oscillatory Fields," Philo. Trans. Royal Soc. London Series A Math., Phys. Eng. Sci. vol. 362, no. 1816, pp. 579-602, March 15, 2004.

[3] L.J. Jiang and A. Ruehli, "On the Frequency Barrier of Surface Integral Equations from a Circuit Point of View," PIERS, Cambridge Jul. 2010

[4] R.F Harrington, Field Computation by Moment Methods, MacMillan, New York, 1968

[5] J.S. Zhao and W.C. Chew, "Integral equation solution of Maxwell's equations from zero frequency to microwave frequencies," IEEE Transactions on Antennas and Propagation, vol. 48, no. 10, pp. 16351645, Oct. 2000.

[6] Z.G. Qian, and W.C. Chew, "Fast full-wave surface integral equation solver for multiscale structure modeling," IEEE Transactions on Antennas and Propagation, AP-57, no. 11, pp. 3594-3601, 2009.

[7] S.M. Rao, D.R. Wilton, and A.W. Glisson, Electromagnetic scattering by surfaces of arbitrary shape, IEEE Trans Antennas Propagat AP-30, 409-418, 1982.

[8] Z.G. Qian and W.C. Chew, "Enhanced A-EFIE with Perturbation Method," UIUC-ECE Research Report No.: CCEML 2-09, August 27, 2009.

[9] M. K. Li and W. C. Chew, "A Domain Decomposition Scheme Based on Equivalence Theorem," Micro. Opt. Tech. Lett., v. 48, no. 9, pp. $1853-$ 1857, Sept. 2006

[10] M. K. Li and W. C. Chew, "Wave-Field Interaction with Complex Structures Using Equivalence Principle Algorithm," IEEE Trans. Antenn Propag., vol. 55, no. 1, pp. 130-138, 2007.

[11] L.E. Sun, W.C. Chew, and J.M. Jin, "Augmented equivalence principle algorithm at low frequencies," Micro. Opt. Tech. Lett., v. 52, no. 10, pp. 2274-2279, Oct. 2010 . 